\title{
Microstructural remodeling in the post-infarct porcine heart measured by diffusion tensor MRI and T1-weighted late gadolinium enhancement MRI
}

\author{
Geoffrey L Kung ${ }^{1,2^{*}}$, Olujimi Ajijola ${ }^{3}$, Rafael J Ramirez ${ }^{3}$, Jin Kyu Gahm, Wei Zhou ${ }^{5}$, N Wisniewski ${ }^{1}$, \\ Aman Mahajan $^{5}$, Alan Garfinkel ${ }^{6}$, Kalyaman Shivkumar $^{3}$, Daniel Ennis ${ }^{1,2}$
}

From 15th Annual SCMR Scientific Sessions

Orlando, FL, USA. 2-5 February 2012

\section{Summary}

The objective of this study was to quantify microstructural remodeling in peri-infarcted and infarcted porcine myocardium using diffusion tensor MRI (DT-MRI) for the first time. High resolution ex vivo late gadolinium enhanced (LGE) MRI was used to segment the DT-MRI data into normal, peri-infarct and infarcted myocardium. LGE-MRI based segmentation produces regions with significantly different microstructural remodeling.

\section{Background}

T1-weighted late gadolinium enhanced (LGE) magnetic resonance imaging (MRI) is recognized as the "gold standard" for MRI based myocardial infarct mapping [1]. It is unclear how variations in LGE signal intensity relate to microstructural remodeling. Diffusion tensor magnetic resonance imaging (DT-MRI) enables 3D evaluation of soft tissue microstructure. Specifically, DT invariants provide a basis for evaluating changes in the trace (TR, magnitude-of-isotropic-diffusion), fractional anisotropy (FA, magnitude-of-anisotropy), and the tensor mode (MD, type-of-anisotropy) as a consequence of remodeling [2]. Previous DT-MRI studies of microstructural remodeling in post-infarct myocardium have not used LGE to segment the remote, peri-infarcted, and infarcted myocardium $[3,4]$. The objective of this study was to use high resolution ex vivo LGE MRI of postinfarct porcine hearts to segment remote, peri-infarcted

'Department of Radiological Sciences, University of California, Los Angeles, Los Angeles, CA, USA

Full list of author information is available at the end of the article and infarcted myocardium and subsequently use this segmentation to quantify microstructural remodeling in peri-infarcted and infarcted myocardium using DT-MRI for the first time.

\section{Methods}

Antero-septal infarctions in adult female porcine hearts $(\mathrm{N}=3)$ were achieved via micro-bead injection distal to the mid left anterior descending coronary artery. After 8 -weeks, Gd-DTPA was injected $(0.1 \mathrm{mmol} / \mathrm{kg})$ and allowed to circulate for 15 minutes before euthanizing. Normal adult porcine hearts $(\mathrm{M}=3)$ served as controls. The hearts were excised and LGE MRI $(0.33 \times 0.33 \times 0.50 \mathrm{~mm}$ resolution) began within 2 hours of sacrifice. Immediately afterwards, co-registered DT-MRI (1x1x3mm resolution) was performed. Myocardial voxels were segmented as normal, peri-infarct or infarct based on signal intensity (SI) thresholds of the LGE images for each heart. We defined bootstrapped histograms and medians with $95 \%$ confidence intervals (95\%-CIs) of each DT invariant in order to make statistical comparisons of non-Gaussian datasets tractable. Remote myocardium in infarcted hearts was also compared to myocardium of control hearts.

\section{Results}

DT invariant medians and 95\%-CIs for segmented myocardium in each heart are listed in Table 1. Invariants in control hearts were similar to remote myocardium in infarcted hearts (Table 1). Intra-heart statistical differences between segmented myocardium were significant. Figure 1 depicts invariant maps for a short axis slice of 
Table 1 Tensor invariant medians and $95 \%$-Cls for normal, remote, peri-infarcted, and infarcted myocardium

\begin{tabular}{|c|c|c|c|c|c|c|}
\hline Heart/region & $\begin{array}{l}\text { Median trace }\left(\mathrm{mm}^{2}\right. \\
/ \mathrm{s})\end{array}$ & $\begin{array}{l}\text { 95\%-Cl of median trace } \\
\left(\mathrm{mm}^{2} / \mathrm{s}\right)\end{array}$ & $\begin{array}{l}\text { Median } \\
\text { FA }\end{array}$ & $\begin{array}{c}95 \%-\mathrm{Cl} \text { of median } \\
\text { FA }\end{array}$ & $\begin{array}{c}\text { Median } \\
\text { mode }\end{array}$ & $\begin{array}{l}95 \%-\mathrm{Cl} \text { of median } \\
\text { mode }\end{array}$ \\
\hline $\begin{array}{l}\text { Control 1: } \\
\text { Normal }\end{array}$ & 0.0017 & {$[0.00170,0.00174]$} & 0.46 & {$[0.453,0.477]$} & 0.76 & {$[0.744,0.781]$} \\
\hline $\begin{array}{l}\text { Control 2: } \\
\text { Normal }\end{array}$ & 0.0014 & {$[0.00140,0.00145]$} & 0.45 & {$[0.437,0.455]$} & 0.72 & {$[0.700,0.743]$} \\
\hline $\begin{array}{l}\text { Control 3: } \\
\text { Normal }\end{array}$ & 0.0017 & {$[0.00166,0.00170]$} & 0.47 & {$[0.462,0.478]$} & 0.71 & {$[0.691,0.740]$} \\
\hline Infarct 1: Remote & 0.0017 & {$[0.00174,0.00176]$} & 0.44 & {$[0.435,0.444]$} & 0.71 & {$[0.699,0.723]$} \\
\hline Infarct 2: Remote & 0.0017 & {$[0.00170,0.00172]$} & 0.45 & {$[0.442,0.450]$} & 0.73 & {$[0.712,0.733]$} \\
\hline Infarct 3: Remote & 0.0020 & {$[0.00194,0.00196]$} & 0.43 & {$[0.430,0.437]$} & 0.72 & {$[0.709,0.726]$} \\
\hline $\begin{array}{l}\text { Infarct 1: Peri- } \\
\text { infarct }\end{array}$ & 0.0019 & {$[0.00186,0.00190]$} & 0.39 & {$[0.380,0.391]$} & 0.62 & {$[0.605,0.643]$} \\
\hline $\begin{array}{l}\text { Infarct 2: Peri- } \\
\text { infarct }\end{array}$ & 0.0020 & {$[0.00200,0.00205]$} & 0.34 & {$[0.332,0.347]$} & 0.62 & {$[0.605,0.642]$} \\
\hline $\begin{array}{l}\text { Infarct 3: Peri- } \\
\quad \text { infarct }\end{array}$ & 0.0023 & {$[0.00225,0.00230]$} & 0.34 & {$[0.334,0.347]$} & 0.62 & {$[0.607,0.645]$} \\
\hline Infarct 1: Infarct & 0.0023 & {$[0.00223,0.00234]$} & 0.28 & {$[0.264,0.288]$} & 0.40 & {$[0.343,0.454]$} \\
\hline Infarct 2: Infarct & 0.0025 & {$[0.00234,0.00254]$} & 0.23 & {$[0.225,0.240]$} & 0.57 & {$[0.539,0.586]$} \\
\hline Infarct 3: Infarct & 0.0025 & {$[0.00247,0.00259]$} & 0.27 & {$[0.265,0.283]$} & 0.55 & {$[0.502,0.572]$} \\
\hline
\end{tabular}

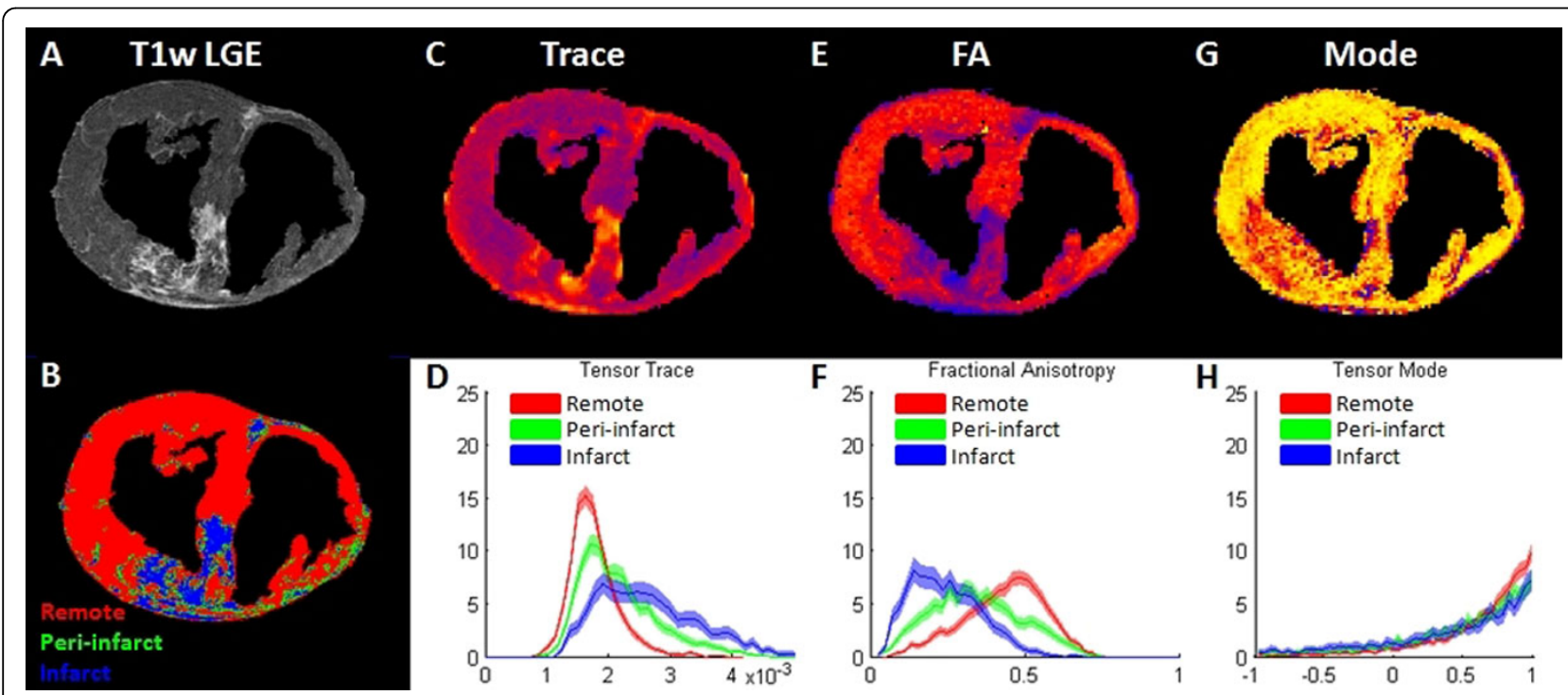

Figure 1 (A) Short-axis T1w LGE image depicting enhancement of the infarct. (B) Segmentation of the T1w LGE slice into remote, peri-infarcted and infarcted myocardium using LGE SI thresholds. Trace map (C) and whole heart segmented bootstrapped histograms (D); FA map (E) and whole heart segmented bootstrapped histograms (F); mode map (G) and whole heart segmented bootstrapped histograms (H).

one heart accompanied by bootstrapped histograms with 95\%-CIs for invariant data for the whole heart. LGE and remote/infarct/peri-infarct segmentation for the same slice are also shown.

\section{Conclusions}

LGE segmentation of DT-MRI data identifies regions of statistically significant microstructural remodeling in peri-infarcted and infarcted myocardium. Improved LGE segmentation methods hold promise for identifying regions of microstructural remodeling when DT-MRI is not available.

\section{Funding}

Mahajan R01-HL084261; Shivkumar R01-HL084261 and R01-HL067647; Garfinkel P01-HL78931 (Core).

\section{Author details}

'Department of Radiological Sciences, University of California, Los Angeles, Los Angeles, CA, USA. ${ }^{2}$ Biomedical Engineering IDP, University of California, Los Angeles, Los Angeles, CA, USA. ${ }^{3}$ Cardiac Arrhythmia Center, David Geffen 
School of Medicine, University of California, Los Angeles, Los Angeles, CA, USA. ${ }^{4}$ Computer Science Department, University of California, Los Angeles, Los Angeles, CA, USA. ${ }^{5}$ Cardiac Anesthesia, Department of Anesthesia, David Geffen School of Medicine, University of California, Los Angeles, Los Angeles, CA, USA. ${ }^{6}$ Cardiovascular Research Laboratory, Department of Cardiology, David Geffen School of Medicine, University of California, Los Angeles, Los Angeles, CA, USA.

Published: 1 February 2012

\section{References}

1. Schelbert EB: Circ CV Imag. 2010, 3(6):743-52.

2. Ennis DB: MRM. 2006, 55(1):136-46.

3. WU Y: JMRI. 2011, 34(1):43-9.

4. Strijkers GJ: NMR Biomed. 2009, 22(2):182-90.

doi:10.1186/1532-429X-14-S1-P66

Cite this article as: Kung et al:: Microstructural remodeling in the postinfarct porcine heart measured by diffusion tensor MRI and T1-

weighted late gadolinium enhancement MRI. Journal of Cardiovascular

Magnetic Resonance 2012 14(Suppl 1):P66.

Submit your next manuscript to BioMed Central and take full advantage of:

- Convenient online submission

- Thorough peer review

- No space constraints or color figure charges

- Immediate publication on acceptance

- Inclusion in PubMed, CAS, Scopus and Google Scholar

- Research which is freely available for redistribution

Submit your manuscript at www.biomedcentral.com/submit 